\section{ORIGINAL RESEARCH}

\author{
Y.S. Lee \\ E.S. Choi \\ C.J. Song
}

\title{
Symptomatic Nerve Root Changes on Contrast- Enhanced MR Imaging after Surgery for Lumbar Disk Herniation
}

\begin{abstract}
BACKGROUND AND PURPOSE: The significance of postoperative nerve root changes (enhancement, thickening, and displacement) is still a topic of debate. The purpose of this study was to evaluate the association between nerve root changes and residual or recurrent symptoms after lumbar surgery with contrast-enhanced MR imaging.
\end{abstract}

\begin{abstract}
MATERIALS AND METHODS: A total of 120 patients with 140 postoperative lumbar disk lesions causing residual or recurrent pain underwent contrast-enhanced MR imaging. The levels at which diskectomies had been performed were retrospectively evaluated for nerve root enhancement (NRE), thickening, and displacement. Association between nerve root changes and corresponding clinical presentations were statistically assessed. Nerve root changes in recurrent disk herniation (RDH) and postoperative epidural fibrosis (PEF) were also evaluated with clinical symptoms.
\end{abstract}

RESULTS: Ninety-two (65.7\%) of the 140 disks demonstrated NRE. Regarding the association with clinical symptoms, the sensitivity was $91.7 \%$, the specificity was $73.2 \%$, the positive predictive value (PPV) was $83.7 \%$, and the negative predictive value was $85.4 \%(P=.000)$. Nerve root thickening and displacement were significantly associated with the clinical symptoms, especially when NRE was combined (PPV, 87.7\% and 87.2\%, respectively). When RDH was combined with all 3 nerve root changes, the PPV was increased up to $94.1 \%$. However, in PEF, the association between nerve root changes and clinical symptoms was not significant unless all 3 nerve root changes were combined.

CONCLUSIONS: In patients with residual or recurrent pain after surgery for lumbar disk herniation, nerve root changes on contrast-enhanced MR imaging were well associated with clinical symptoms, of which NRE was the most significant finding.

$\mathbf{M}_{\mathrm{r}}^{\mathrm{R}}$ $\mathrm{R}$ imaging is usually performed to investigate the causes of residual or recurrent symptoms after lumbar disk surgeries. Specifically, contrast-enhanced MR imaging studies have been used to assist in the differentiation of postoperative epidural fibrosis (PEF) from recurrent disk herniation (RDH). ${ }^{1-3}$ However, symptomatic nerve root changes such as nerve root enhancement (NRE), thickening, and displacement can be seen when there is radicular pain even without $\mathrm{RDH}$ or PEF. The clinical significance of postoperative nerve root changes is still being debated. A few studies have investigated nerve root signs on postoperative MR imaging or associations between such signs and clinical outcomes, and the results are contradictory. ${ }^{2-8}$ Abnormal enhancement of nerve roots on MR imaging has been determined to be well correlated with clinical symptoms in patients who have not undergone spinal surgery. ${ }^{9-11}$ However, the clinical significance of NRE on postoperative lumbar spine MR imaging has not yet been definitively determined. It has generally been accepted that nerve root thickening by itself has no diagnostic value, ${ }^{2-4}$ whereas the combination of thickening and enhancement in the nerve root correlates with symptoms ${ }^{7}$ or early recurrence of symptoms, particularly when associated with $\mathrm{RDH} .^{2}$ The

Received October 10, 2008; accepted December 9.

From the Departments of Radiology (Y.S.L.), and Rehabilitation Medicine (E.S.C.), Daejeon St. Mary's Hospital, The Catholic University of Korea, Daejeon, Korea; and Department of Radiology (C.J.S.), Chungnam National University School of Medicine, Daejeon, Korea.

Please address correspondence to Yeon Soo Lee, MD, Department of Radiology, Daejeon St. Mary's Hospital, The Catholic University of Korea, Daejeon, Korea, 520-2, Deaheungdong, Jung-gu, Daejeon 301-723, Korea; e-mail: yslee1074@medimail.co.kr

Indicates open access to non-subscribers at www.ajnr.org

DOI 10.3174/ajnr.A1497 purpose of this study was to evaluate the association between nerve root changes and clinical symptoms such as residual or recurrent sciatica after lumbar disk surgery with use of contrast-enhanced MR imaging.

\section{Materials and Methods}

\section{Subjects}

We retrospectively reviewed patients who had undergone MR imaging studies because of residual or recurrent sciatica after surgeries for disk herniation at lumbosacral levels in our university hospital from 1998 to 2006. Patients who had concurrent lumbosacral stenosis from spondylolisthesis, arachnoiditis, and postoperative infection were not included in this study. Also, patients who had only axial low back pain without radicular pain and who had incomplete medical information, including the typical symptoms and signs suggesting the level of radiculopathy, were also excluded. Among the patients with postoperative sciatica, 120 patients ( 70 men and 50 women; age range, 17-80 years; mean age, 48.5 years) with 140 postoperative lumbar disk lesions were recruited. The disk levels where operations had been done were L3-L4 in 12 cases, L4-L5 in 92 cases, and L5-S1 in 36 cases. The time from the surgery to postoperative MR imaging ranged from 8 days to 180 months (mean, 50 months). Twenty-four disk lesions were examined less than 6 months after surgery, whereas 116 disks were studied 6 months after surgery.

\section{MR Imaging Protocol}

We obtained postoperative images using MR imaging scanners of 1.5T (Intera; Philips Medical Systems, Best, the Netherlands) or 0.5T (Gyroscan T5; Philips, Eindhoven, the Netherlands). Fifty-seven cases were examined with the $1.5 \mathrm{~T}$ unit and 83 with the $0.5 \mathrm{~T}$ instrument. All patients were studied with the following imaging sequences 
before administration of contrast material: turbo spin-echo axial T1weighted imaging (TR, $550 \mathrm{~ms}$; TE, $25 \mathrm{~ms}$ ) with a 4-mm section thickness and a 0.4-mm interspace, and turbo spine echo sagittal T1weighted image (TR, $740 \mathrm{~ms}$; TE, $13 \mathrm{~ms}$ ) with a 4-mm section thickness and a 0.4-mm interspace. In all patients, axial T1-weighted image and fat-suppressed sagittal T1-weighted image sequences were repeated after intravenous administration of $0.1 \mathrm{mmol} / \mathrm{kg}$ of gadolinium-DTPA (Magnevist; Bayer Schering Pharma, Pittsburgh, Pa). The FOV was $220 \times 220 \mathrm{~mm}$ for axial images and $320 \times 320 \mathrm{~mm}$ for sagittal images. The imaging matrix size was $256 \times 205$ in axial images and $512 \times 256$ in sagittal images.

\section{MR Imaging Analysis}

The levels at which diskectomies had been performed were evaluated for NRE, thickening, and displacement. Two radiologists (Y.S.L. and C.J.S.) were blinded to the patient's medical history and were unaware of the exact level of radiculopathy.

Measurement of Nerve Root Enhancement. NRE was divided into intradural and extradural forms and was assessed in 2 ways: by comparing precontrast and postcontrast axial T1-weighted images and by comparing the enhanced nerve root to the corresponding nerve root on the contralateral side. ${ }^{7}$ Enhancement was evaluated as present or absent. When intradural NRE was seen, the degree of enhancement was measured on the axial T1-weighted image in a 2.4$\mathrm{mm}^{2}$ region of interest. The maximal pixel value in the region of interest was compared with that of the corresponding region of interest on the precontrast image, thus allowing calculation of a difference ratio 1 (postcontrast/precontrast maximum pixel value in the affected nerve root). The same procedure was used for comparison with the postcontrast contralateral nerve root, calculating the difference ratio 2 (enhanced affected nerve root/enhanced contralateral nerve root). Intradural NRE was considered present only if both the difference ratios were more than 1.2. Meanwhile, extradural NRE was assessed visually because of the partial volume effect from the enhanced dura or scar tissue in close contact with the nerve root. Visual assessment was performed by 2 radiologists with more than 10 years of experience. In cases in which the observers disagreed, pixel measurements were performed.

NRE correlated with clinical symptoms. On review of the medical record of each subject, the distribution of typical radicular pain, presence of myotomal and/or dermatomal deficits as well as loss of tendon stretch reflex of the quadriceps and triceps surae muscles were used to define the level of radiculopathy. In addition, some of our subjects had undergone electromyographic studies, and the results were also used to determine the involved root level. The association between NRE and clinical symptoms was determined when the site of NRE at the previous surgery was matched with the level of radiculopathy from typical neurologic signs and/or electromyographic studies.

Nerve Root Thickening. Thickening of a nerve root was defined as a more than $50 \%$ increase in diameter compared with the contralateral nerve root at the same level. ${ }^{8}$ To avoid misinterpretation because of adjacent scar tissues, nerve root thickening was assessed only on postcontrast images. Thickening of nerve roots was also associated with clinical symptoms.

Nerve Root Displacement. Nerve root displacement was defined as present if the position of the nerve root was different from what was expected, comparing with the position of the contralateral nerve root at the same level. Nerve root displacement was correlated with clinical symptoms as well.

\begin{tabular}{|c|c|c|c|c|}
\hline \multicolumn{5}{|c|}{$\begin{array}{l}\text { Table 1: Relationship between nerve root enhancement and } \\
\text { postoperative sciatica }\end{array}$} \\
\hline \multicolumn{5}{|l|}{ Nerve Root } \\
\hline \multirow[t]{2}{*}{ Enhancement } & \multicolumn{3}{|c|}{ Clinical Symptom } & \multirow[t]{2}{*}{$P$ value } \\
\hline & Matched & Unmatched & Total & \\
\hline Present & 77 & 14 & 92 & $.000^{*}$ \\
\hline Absent & 7 & 41 & 48 & \\
\hline Total & 84 & 56 & 140 & \\
\hline
\end{tabular}

* Value is statistically significant using the $\chi^{2}$ test $(P<.05)$.

Recurrent Disk Herniation and Postoperative Epidural Fibrosis. $\mathrm{RDH}$ was defined as a polypoid focal low signal intensity mass at the operative site on T1-weighted image with no central enhancement, which was contiguous with the parent disk unless sequestrated. $\mathrm{RDH}$ was classified as protrusion, extrusion, and sequestered disk according to the American Society for Neuroradiology consensus nomenclature. ${ }^{12,13}$ If RDH was combined with PEF, the case was enrolled into the RDH category.

On the other hand, PEF was defined as epidural soft tissue at the operative site with consistent contrast enhancement. The amount of peridural scarring was graded on a scale of 0 to 4 in each quadrant according to the method of Ross et al. ${ }^{14}$ In this system, scale 0 is no scarring, scale 1 is a quadrant with $0 \%$ to $25 \%$ scar fill, scale 2 is $25 \%$ to $50 \%$, scale 3 is $50 \%$ to $75 \%$, and scale 4 is $75 \%$ to $100 \%$, respectively. We determined the correlations between scar grade, nerve root changes, and clinical symptoms. The association among the nerve root changes of RDH or PEF and clinical symptoms was also assessed, respectively.

\section{Statistical Analysis}

We determined the frequency analyses of the nerve root changes with regard to the clinical symptoms by using the $\chi^{2}$ test (version 14.0; SPSS, Chicago, Ill). Associations between the nerve root changes in $\mathrm{RDH}$ or PEF and clinical symptoms were also analyzed. Statistical significance was defined as $P<.05$.

\section{Results}

\section{Nerve Root Enhancement and Clinical Symptoms}

Among all 140 disks, 92 (65.7\%) demonstrated NRE. Frequency analysis revealed that the site of NRE was significantly associated with the corresponding level of radiculopathy in the subject, showing a sensitivity of $91.7 \%$, a specificity of $73.2 \%$, thus, a positive predictive value (PPV) of $83.7 \%$ and a negative predictive value of $85.4 \%(P=.000$; Table 1$)$.

Among the 92 disks with NRE, 35 cases were intradural type (Fig 1) and 70 cases were extradural type. Thirteen cases showed combined intradural and extradural enhancement (Fig 2). Good associations with clinical symptoms were seen in both types, with the PPV of $85.7 \%$ in intradural form and $85.7 \%$ in extradural form.

\section{Time between Surgery and Postoperative MR Imaging}

The time between disk surgeries and postoperative MR imaging studies was divided into "before 6 months" and "after 6 months." Twenty-four cases of all 140 disks belonged to the former group (mean, 2.9 months), and 18 of them showed NRE. In 10 of 18 NRE cases, the sites of NRE agreed with the clinical symptoms but no statistical significance $(P=.346)$. Meanwhile, 116 cases were studied after 6 months (mean, 68.2 

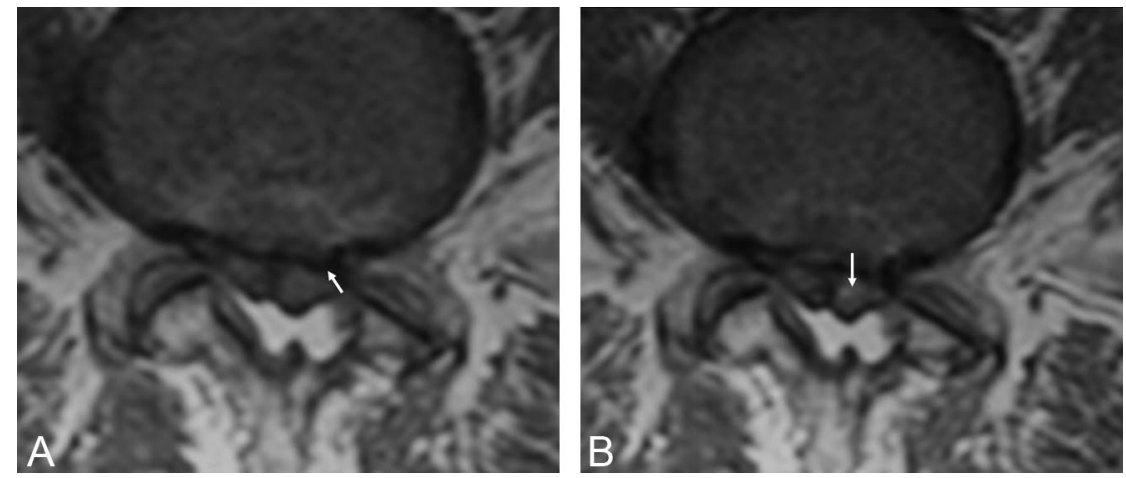

Fig 1. A 53-year-old man who underwent an operation 15 years ago. A, Precontrast axial T1-weighted MR image shows left subarticular recurrent disk herniation at L4-L5 level (arrow) $B$, Postcontrast axial T1-weighted MR image shows contrast enhancement in the left intradural nerve root (arrow).
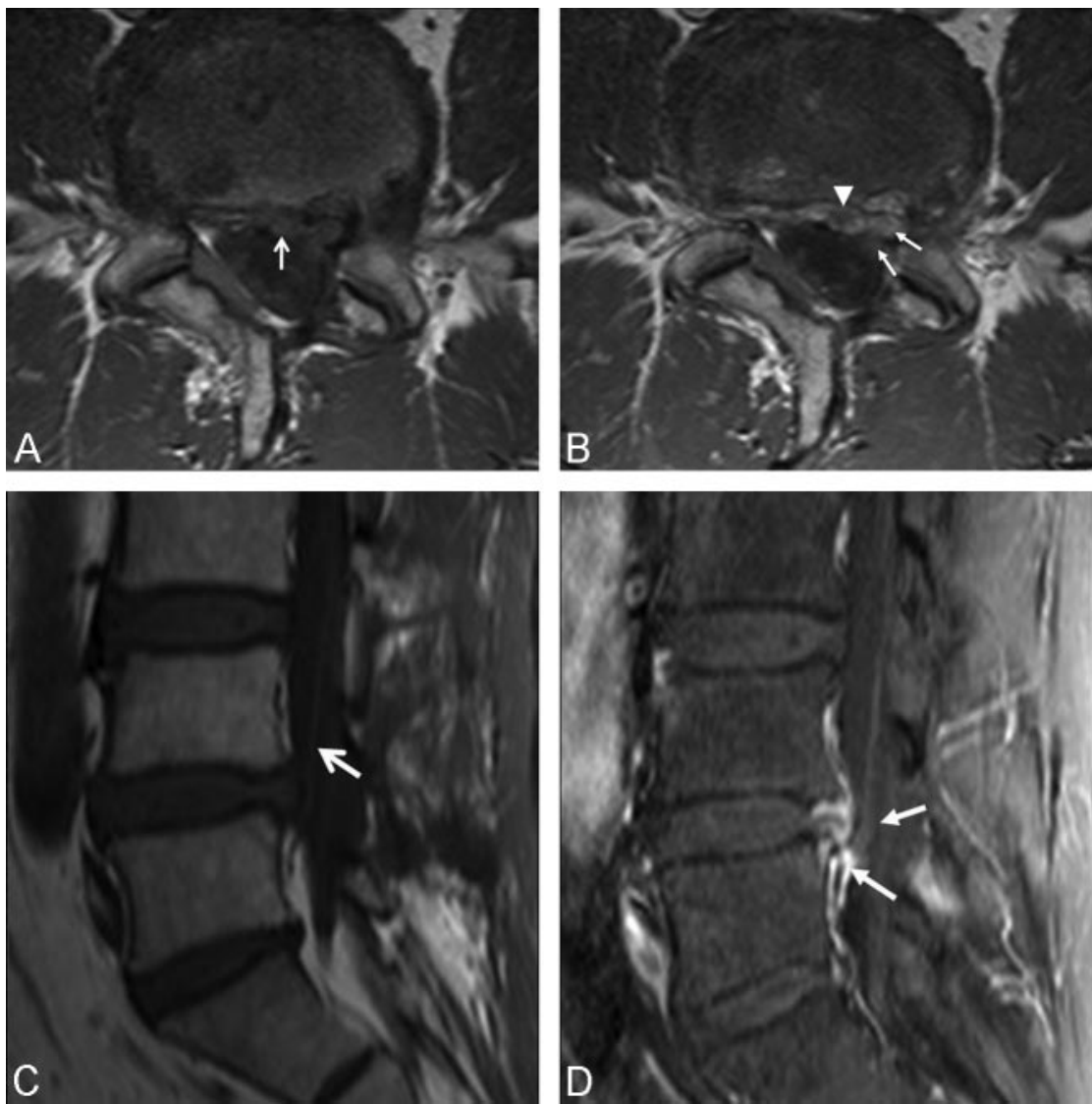

Fig 2. A 63-year-old man with left $L 5$ radiculopathy on the electromyographic study, who underwent an operation 12 months ago. $A$, Axial T1-weighted MR image before contrast injection shows left central intermediate signal intensity epidural lesion (arrow). B. Axial T1-weighted MR image after contrast injection. Enhancement is noted in the left epidural and intradural L5 nerve root (arrows). Nonenhanced disk protrusion is seen (arrowhead). Nonenhanced sagittal T1-weighted (C) and enhanced sagittal T1-fat suppressed (D) MR images show mildly enhanced intradural nerve root and bright enhancement of epidural nerve root (arrows).

months); of these cases, 72 disks showed NRE. In 63 of $72 \mathrm{NRE}$ cases, the sites of NRE were significantly associated with clinical symptoms $(P=.000)$.

\section{Nerve Root Thickening and Clinical Symptoms}

Nerve root thickening was seen in 76 of all 140 disks, and 55 cases $(72.4 \%)$ showed good association with clinical symptoms $(P=.001)$. In those 76 disks with nerve root thickening, $57(75 \%)$ were combined with NRE, and 50 cases $(87.7 \%)$ with combined pathologic features showed significant association with symptoms $(P=.000)$, with a PPV of $87.7 \%$.

\section{Nerve Root Displacement and Clinical Symptoms}

Nerve root displacement was seen in 64 of all 140 disks, of which the causes were RDH in 34 cases and PEF in 30 cases. Forty-seven cases $(73.4 \%)$ showed good association with clinical symptoms $(P=.003)$. In those 47 disks with nerve root displacement, 41 cases $(87.2 \%)$ were combined with NRE. The disks with combined pathologic features showed significant association with symptoms $(P=.000)$, with a PPV of $87.2 \%$.

\section{RDH and Nerve Root Changes}

In $39 \mathrm{RDH}$ cases consisting of 19 protrusions, 19 extrusions, and 1 sequestered disk, NRE was observed in 33 cases (84.6\%). 

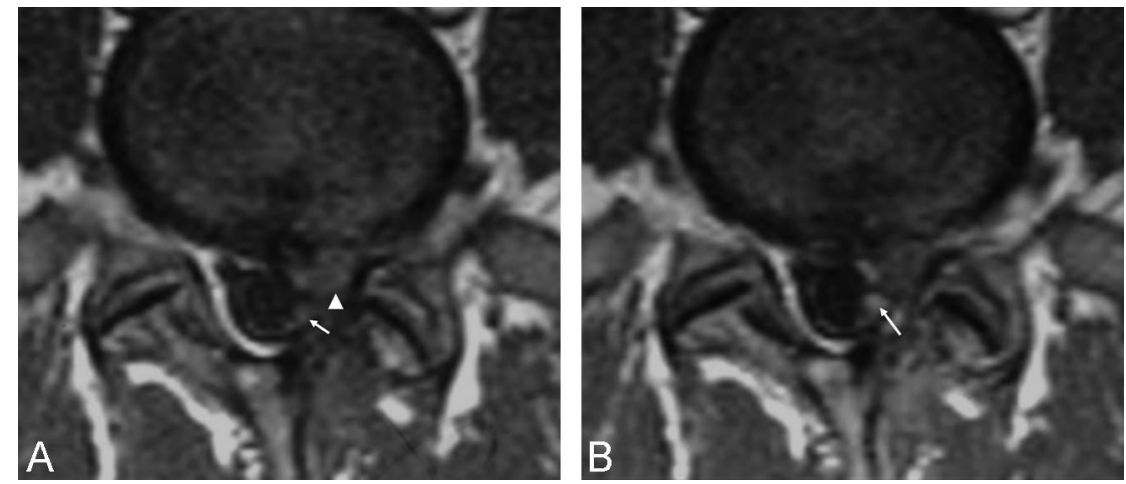

Fig 3. A 27-year-old man with recurrent disk herniation confirmed by reoperation. $A$, Precontrast axial T1- weighted MR image at L5-S1 level shows left subarticular recurrent disk extrusion with intermediate signal intensity (arrowhead). Thickened and posteriorly displaced left intradural nerve root (arrow) is noted. B, Postcontrast axial T1-weighted MR image reveals nonenhanced recurrent disk herniation. A combination of nerve root enhancement, thickening, and displacement (arrow) is also seen.

$\begin{aligned} & \text { Table 2: Positive predictive values between nerve root changes and } \\
& \text { clinical symptoms in recurrent disk herniation of the lumbosacral } \\
& \text { spine }\end{aligned}$
\begin{tabular}{lcc} 
Nerve Root & $\begin{array}{c}\text { Positive Predictive } \\
\text { Value (\%) }\end{array}$ & $P$ value \\
Changes & 87.9 & $.000^{*}$ \\
\hline Enhancement & 85.2 & $.003^{*}$ \\
Thickening & 84.4 & $.001^{*}$ \\
Displacement & 94.1 & $.002^{*}$ \\
Combination of all
\end{tabular}

*Values are statistically significant using the $\chi^{2}$ test $(P<.05)$.

In $29 \mathrm{RDH}$ cases with NRE (87.9\%), there was significant association with clinical symptoms $(P=.0000)$. Nerve root thickening $(n=27)$ and displacement $(n=32)$ were also seen in $\mathrm{RDH}$, showing good association with clinical symptoms in $85.2 \%$ and $84.4 \%$ of them, respectively. When a $\mathrm{RDH}$ was combined with all 3 nerve root changes (Fig 3), the PPV of the MR image in diagnosing the level of radiculopathy further increased up to $94.1 \%$ (Table 2).

\section{PEF and Nerve Root Changes}

PEF was seen in 84 (60\%) of 140 disks, consisting of 30 cases in first-degree changes, 30 cases in second-degree changes, and 24 cases in third-degree changes. NRE was seen in 58 of them (69\%). In 58 cases of PEF with NRE, 40 cases agreed with the clinical symptoms but with no significance $(P=.069$; Fig 4$)$. The amount of PEF did not correlate significantly with symptoms. Neither nerve root thickening nor displacement was associated with clinical symptoms. However, in 14 PEF cases with all 3 nerve root changes, 13 cases showed significant association with clinical symptoms $(P=.008$; Table 3$)$.

\section{Discussion}

NRE on MR imaging studies showed good correlation with the clinical symptoms in patients with lumbar disk herniation who had not had spinal surgery. . $^{20,11,15,16}$ In such studies, NRE was determined to be a good indicator of the presence of clinical symptoms and was thought to indicate the affected nerve root. Recently, the causes of recurrent pain in patients after lumbar disk surgery have been reported in some studies including contrast-enhanced MR imaging, ${ }^{4,6,17,18}$ but the association between the site of the nerve root changes such as enhancement, thickening, displacement, and radicular pain is not well defined yet. ${ }^{4,9,17,19}$ We evaluated the association be- tween nerve root changes and residual or recurrent symptoms after lumbar spine surgery by using contrast-enhanced MR imaging.

Some of our cases were studied on $0.5 \mathrm{~T}$ and the others, on 1.5T MR imaging, in which circumstance a difference in the image resolution for the evaluation of the nerve root changes could be possible. In recent years, some reports have described on MR imaging of the spine at $3 \mathrm{~T} .{ }^{20,21}$ It appears that $3 \mathrm{~T}$ imaging, by virtue of its improved signal-to-noise ratio and resultant improved spatial resolution, can be more successful to evaluate the spine and its contents, as normal and pathologic structures should be delineated better. Current practical limitations, however, made it difficult to identify some lesions (eg, subtle enhancing lesions) in the spine. Therefore, the use of contrast agents and the need for possible adjustments of their respective dosages for contrast-enhanced $3 \mathrm{~T}$ spinal imaging

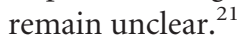

The most common technique used to evaluate NRE on MR images is visual comparison between the precontrast and postcontrast images and the affected and unaffected nerve roots. ${ }^{7}$ In our study, intradural NRE was determined to be present only if both the difference ratios were more than 1.2 (ie, postcontrast/precontrast maximal pixel value in the affected nerve root and enhanced affected nerve root/enhanced contralateral nerve root). Grane and Lindqvist ${ }^{7}$ also used the maximal pixel value of the region of interest instead of the mean value because of the small sizes of the nerve structures. To evaluate extradural NRE, 2 radiologists used visual comparison because partial volume effects from enhanced scar tissue close to the nerve root often made pixel measurements inaccurate. If an enhanced nerve root could not be differentiated from RDH or PEF, a comparison with the unaffected nerve root was performed. We did not use fat-suppressed axial images to evaluate NRE because abnormal postoperative NRE may be more difficult to differentiate from the normal slight pial-root enhancement usually seen on fat-suppressed images. ${ }^{18}$

In this study, 92 cases $(65.7 \%)$ of 140 postoperative disks demonstrated NRE, and the sites of root enhancement were well matched to the level of radiculopathy, with a PPV of $83.7 \%$. The mechanism of abnormal NRE on MR images has been hypothesized to be a breakdown in the blood-nerve barrier caused by injury, ischemia, inflammation, demyelination, or axonal degeneration, and increased vascular permeability with accompanying vasodilation. ${ }^{6,10}$ The relationship between 

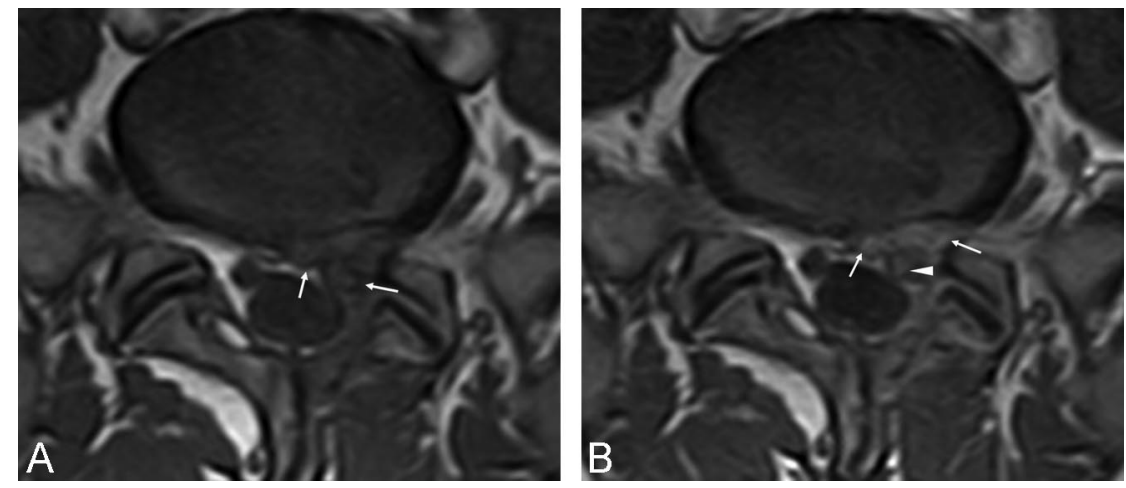

Fig 4. A 21 -year-old man with recurrent left $\mathrm{S} 1$ sciatica and radiculopathy by electromyography. $A$, Precontrast axial T1-weighted MR image reveals an intermediate signal intensity lesion in the left epidural space including an ill-defined margin of the left S1 nerve root (arrows). B, Postcontrast axial T1-weighted image shows enhancement of left epidural mass, suggesting postoperative fibrosis (arrows) and focal enhancement of the thickened left epidural S1 nerve root (arrowhead).

\begin{tabular}{lcc}
\hline $\begin{array}{l}\text { Table 3: Positive predictive values between nerve root changes and } \\
\text { clinical symptoms in postoperative epidural fibrosis }\end{array}$ \\
\hline $\begin{array}{l}\text { Nerve Root } \\
\text { Changes }\end{array}$ & $\begin{array}{c}\text { Positive Predictive } \\
\text { Value (\%) }\end{array}$ & $P$ value \\
\hline Enhancement & 69.0 & .069 \\
Thickening & 67.4 & .231 \\
Displacement & 63.0 & .726 \\
Combination of all & 92.9 & $.008^{*}$ \\
\hline
\end{tabular}

* Value is statistically significant using the $\chi^{2}$ test $(P<.05)$.

blood-nerve barrier disruption and radicular pain has been studied. Taneichi et $\mathrm{a}^{19}$ showed that NRE corresponds to intraneural edema causing radicular pain rather than neurologic deficits. The nerve root becomes inflamed as a result of changes in the intraneural pressure, exposure to chemical irritants, or ischemia, and even minor mechanical deformation may lead to radicular pain. ${ }^{15}$ In a study by Vroomen et al, ${ }^{22}$ root enhancement was found to correlate with sensory loss, irrespective of whether a herniated disk compressed the nerve root. This would make NRE a sign of a radicular pathologic disorder in general, rather than a sign of a root pathologic disorder because of disk compression.

In 92 disks with NRE, both intradural and extradural types had good associations with clinical symptoms, with a high PPV of $86 \%$. Grane and Lindqvist ${ }^{7}$ also reported a good correlation between nerve root data and clinical symptoms in $59 \%$ of patients with intradural enhancement and in $84 \%$ of cases with focal enhancement.

The relationship between postoperative MR imaging study time and NRE has been of clinical interest. ${ }^{23-25}$ NRE in the immediate postoperative period may be a normal finding. ${ }^{3}$ This phenomenon has been reported in $29 \%$ to $62 \%$ of asymptomatic patients 3 to 6 weeks after surgery, and the incidence of NRE decreased by 3 months with no enhancement after 6 months. ${ }^{23,24}$ Enhancement 6 months after surgery is considered pathologic. ${ }^{23,25}$ Jinkins et $\mathrm{al}^{6}$ observed a good correlation between NRE beyond 6 to 8 months postoperatively and clinical symptoms. In our study, although the association between NRE ( $n=18)$ before 6 months postsurgery and clinical symptoms was not significant, $87.5 \%$ of $72 \mathrm{NRE}$ cases 6 months postoperatively showed significant association with clinical symptoms.

The swollen nerve root is easily compressed in the narrow root sheath, leading to severe sciatica. ${ }^{26}$ In our study, $72 \%$ of disks with nerve root thickening $(n=76)$ had good association with symptoms, and when combined with NRE, the PPV significantly increased. Grane and Lindqvist ${ }^{7}$ also reported that a combination of nerve root thickening and enhancement correlated with symptoms in $86 \%$ of patients, though no correlation between recurrent symptoms and the occurrence of thickened nerve roots only was found in their previous study. ${ }^{8}$

In our study, $73 \%$ of disks with nerve root displacement $(n=64)$ showed good association with clinical symptoms, and when combined with NRE, the PPV was $87 \%$. In a displaced nerve root resulting from disk herniation, the nerve root can be pushed and compressed or retracted, impeding nutritional transport to the nerve root, thus producing secondary radicular pain. ${ }^{2,27,28}$ However, in the study of Nygaard et al, ${ }^{4}$ no association between nerve root changes and clinical outcome was found when patients with RDH were excluded.

$\mathrm{RDH}$ and PEF are definitely of clinical importance in postoperative low back pain syndromes. ${ }^{2,3,18,29}$ Although there are some controversies regarding the correlation between the presence of enhancing scar and the patient's symptoms, ${ }^{30}$ Ross et $\mathrm{al}^{14}$ reported on 197 patients with extensive peridural scar, identifying that they were 3.2 times more likely to experience recurrent radicular pain. BenDebba et $\mathrm{al}^{31}$ demonstrated a significant association between extensive epidural scarring and activity-related pain. On the contrary, there have also been reports that clearly demonstrate the presence of scar formation in asymptomatic patients. ${ }^{32,33}$ In addition, symptomatic patients had the same amount of scar tissue on MR imaging as had asymptomatic patients, more than 1 year after surgery. ${ }^{7}$ In most cases, epidural scar tissue without disk herniation seems to be unimportant in recurrent sciatica, suggesting that disk herniation is probably the main cause of such sciatica in symptomatic postoperative patients. However, disk herniation may be found in asymptomatic patients after lumbar diskectomy also. Therefore, Grane ${ }^{2}$ claimed that it was important to evaluate the agreement between the recurrent pain and the location of disk herniation and to exclude other painful pathologic changes. In our study, NRE was observed in $85 \%$ of RDH cases $(n=39)$, and each nerve root change in them had significant associations with clinical symptoms. When an RDH was combined with all 3 nerve root changes, the PPV was highest. Meanwhile, NRE was observed in $69 \%$ of PEF $(n=84)$, and only PEF cases combined with all nerve root changes showed significant association with clinical symptoms. 
Our study had some limitations. Because of a retrospective study design, we did not evaluate the preoperative lumbar spine MR images in all subjects, so we could not compare preoperative and postoperative nerve root changes. Another limitation was that investigation of the nerve root changes in asymptomatic postoperative cases had not been made.

\section{Conclusions}

In patients with residual or recurrent sciatica after surgery for lumbar disk herniation, nerve root changes, especially root enhancement on contrast-enhanced MR imaging 6 months postoperatively showed the best association with clinical symptoms. When an RDH was combined with all 3 nerve root changes, the PPV of the MR image could be best to diagnose the involved nerve root.

\section{References}

1. Duda JJ Jr, Ross JS. The postoperative lumbar spine: imaging considerations. Semin Ultrasound CT MRI 1993;14:425-36

2. Grane P. The postoperative lumbar spine. A radiological investigation of the lumbar spine after discectomy using MR imaging and CT. Acta Radiol Suppl 1998;414:1-23

3. Babar S, Saifuddin A. MRI of the post-discectomy lumbar spine. Clin Radiol 2002;57:969-81

4. Nygaard OP, Jacobsen EA, Solberg T, et al. Nerve root signs on postoperative lumbar MR imaging. A prospective cohort study with contrast enhanced MRI in symptomatic and asymptomatic patients one year after microdiscectomy. Acta Neurochir (Wien) 1999;141:619-22, discussion 623

5. Yukawa Y, Kato F, Kajino G, et al. Serial gadolinium-enhanced MR imaging after lumbar disc resection: observation of the affected root. J Spinal Disord 1997;10:404-09

6. Jinkins JR, Osborn AG, Garrett D, et al. Spinal nerve enhancement with Gd-DTPA: MR correlation with the postoperative lumbosacral spine. AJNR Am J Neuroradiol 1993;14:383-94

7. Grane $P$, Lindqvist $M$. Evaluation of the post-operative lumbar spine with $M R$ imaging. The role of contrast enhancement and thickening in nerve roots. Acta Radiol 1997;38:1035-42

8. Grane P, Tullberg T, Rydberg J, et al. Postoperative lumbar MR imaging with contrast enhancement. Comparison between symptomatic and asymptomatic patients. Acta Radiol 1996;37:366-72

9. Georgy BA, Snow RD, Hesselink JR. MR imaging of spinal nerve roots: techniques, enhancement patterns, and imaging findings. AJR Am J Roentgenol 1996;166:173-77

10. Jinkins JR. MR of enhancing nerve roots in the unoperated lumbosacral spine. AJNR Am J Neuroradiol 1993;14:193-202

11. Toyone T, Takahashi K, Kitahara H, et al. Visualisation of symptomatic nerve roots. prospective study of contrast-enhanced MRI in patients with lumbar disc herniation. J Bone Joint Surg Br 1993;75:529-33

12. Costello RF, Beall D. Nomenclature and standard reporting terminology of intervertebral disk herniation. Magn Reson Imaging Clin N Am 2007;15:167-74

13. Fardon DF, Milette PC. Nomenclature and classification of lumbar disc pathology. Recommendations of the Combined Task Forces of the North Amer- ican Spine Society, American Society of Spine Radiology, and American Society of Neuroradiology. Spine 2001;26:E93-E113

14. Ross JS, Robertson JT, Frederickson RC, et al. Association between peridural scar and recurrent radicular pain after lumbar discectomy: magnetic resonance evaluation. Neurosurgery 1996;38:855-61

15. Tyrrell PN, Cassar-Pullicino VN, McCall IW. Gadolinium-DTPA enhancement of symptomatic nerve roots in MRI of the lumbar spine. Eur Radiol 1998;8:116-22

16. Kobayashi S, Yoshizawa H, Hachiya Y, et al. Vasogenic edema induced by compression injury to the spinal nerve root. Distribution of intravenously injected protein tracers and gadolinium-enhanced magnetic resonance imaging. Spine 1993;18:1410-24

17. Tullberg T, Grane P, Isacson J. Gadolinium-enhanced magnetic resonance imaging of 36 patients one year after lumbar disc resection. Spine 1994;19:176-82

18. Von Goethem JW, Parizel PM, Jinkins JR. Review article: MRI of the postoperative lumbar spine. Neuroradiology 2002;44:723-39

19. Taneichi H, Abumi K, Kaneda K, et al. Significance of Gd-DTPA-enhanced magnetic resonance imaging for lumbar disc herniation: the relationship between nerve root enhancement and clinical manifestations. J Spinal Disord 1994; 7:153-60

20. Shapiro MD. MR imaging of the spine at 3 T. Magn Reson Imaging Clin N Am 2006;14:97-108

21. Phalke VV, Gujar S, Quint DJ. Comparison of 3.0 T versus 1.5 T MR: imaging of the spine. Neuroimaging Clin N Am 2006;16:241-48

22. Vroomen PC, Van Hapert SJ, Van Acker RE, et al. The clinical significance of gadolinium enhancement of lumbar disc herniations and nerve roots on preoperative MRI. Neuroradiology 1998;40:800-06

23. Van de Kelft EJ, Van Goethem JW, De La Porte C, et al. Early postoperative gadolinium-DTPA-enhanced MR imaging after successful lumbar discectomy. Br J Neurosurg 1996;10:41-49

24. Floris R, Spallone A, Aref TY, et al. Early postoperative MRI findings following surgery for herniated lumbar disc. Part II: A gadolinium-enhanced study. Acta Neurochir (Wien) 1997;139:1101-07

25. Henk CB, Brodner W, Grampp S, et al. The postoperative spine. Top Magn Reson Imaging 1999;10:247-64

26. Takata K, Inoue S, Takahashi K, et al. Swelling of the cauda equina in patients who have herniation of a lumbar disc. A possible pathogenesis of sciatica. J Bone Joint Surg Am 1988;70:361-68

27. Rydevik B, Brown MD, Lundborg G. Pathoanatomy and pathophysiology of nerve root compression. Spine 1984;9:7-15

28. Pfirrmann CW, Dora C, Schmid MR, et al. MR image-based grading of lumbar nerve root compromise due to disk herniation: reliability study with surgical correlation. Radiology 2004;230:583-88

29. Saunders WP, Truumees E. Imaging of the postoperative spine. Semin Ultrasound CT MRI 2004;25:523-35

30. Coskun E, Süzer T, Topuz O, et al. Relationships between epidural fibrosis, pain, disability, and psychological factors after lumbar disc surgery. Eur Spine J 2000;9:218-23

31. BenDebba M, Augustus van Alphen H, Long DM. Association between peridural scar and activity-related pain after lumbar discectomy. Neurol 1999;21 Suppl 1:S37-42

32. Almeida DB, Prandini MN, Awamura Y, et al. Outcome following lumbar disc surgery: the role of fibrosis. Acta Neurochir (Wien) 2008;150:1167-76

33. Annertz M, Jonsson B, Stromqvist B, et al. No relationship between epidural fibrosis and sciatica in the lumbar postdiscectomy syndrome. A study with contrast-enhanced magnetic resonance imaging in symptomatic and asymptomatic patients. Spine 1995;20:449-53 